\section{Factors associated with erythropoiesis-stimulating agent hyporesponsiveness anemia in chronic kidney disease patients}

\author{
Kamalas Amnuay, ${ }^{1}$ Nattachai Srisawat, ${ }^{2}$ \\ Kitsada Wudhikarn, ${ }^{3,4}$ \\ Thamathorn Assanasen, 5 \\ Chantana Polprasert ${ }^{3,4}$ \\ ${ }^{1}$ Department of Medicine, ${ }^{2}$ Division of \\ Nephrology, ${ }^{3}$ Division of Hematology, \\ Department of Medicine, Faculty of \\ Medicine,Chulalongkorn University, \\ King Chulalongkorn Memorial Hospital, \\ Bangkok; ${ }^{4}$ Research Collaborations in \\ Hematologic Malignancies and \\ Hematopoietic Stem Cell \\ Transplantation, Chulalongkorn \\ University, Bangkok; ${ }^{5}$ Department of \\ Pathology, Chulalongkorn University, \\ King Chulalongkorn Memorial Hospital, \\ Bangkok, Thailand
}

\section{Abstract}

Anemia is one of the most common problems in chronic kidney disease (CKD). Despite comprehensive investigations in several cases, definite causes of anemia frequently remain unknown. Our study aimed to analyze the factors that possibly affect anemia in CKD patients who were referred for hematology consultation. A total of 87 patients were retrospectively included in the cohort. Forty-four cases were excluded, including 30 cases with unavailable intact parathyroid hormone (iPTH) data, 11 cases with bone marrow diseases ( 8 Pure red cell aplasia, 3 Myelodysplastic syndrome) and 3 cases with thalassemia. In total, 43 patients were analyzed. Patients with high iPTH had a significantly lower Hemoglobin ( $\mathrm{Hb}$ ) level and required a higher dose of erythropoiesis stimulating agents (ESAs) compared with the normal iPTH group (Hb 8.29 vs 9.24 $\mathrm{mg} / \mathrm{dL}, \quad \mathrm{P}=0.032$ and ESAs dose of 16,352.94 vs. 12,444.44 U/week, $\mathrm{P}=0.024)$. Univariate, followed by stepwise multivariate analysis was performed and determined that serum phosphate (PO4) was significantly associated with lower $\mathrm{Hb}$ level $(\mathrm{P}=0.01$ and $\mathrm{P}=0.013$, respectively). In addition, $\mathrm{Hb}$ level was inversely correlated with $\mathrm{iPTH}$ and serum phosphate (PO4) level ( $\mathrm{r}=-0.54$, $\mathrm{P}<0.001$ and $\mathrm{r}=-0.47, \mathrm{P}=0.005$; respective- ly). Mineral disequilibrium is an important factor associated with anemia in ESA hyporesponsive CKD. Also, hyperphosphatemia and secondary hyperparathyroidism are significantly correlated with low $\mathrm{Hb}$. As a result, we strongly suggest correction of mineral disequilibrium factors prior to performing bone marrow study.

\section{Introduction}

Anemia in chronic kidney disease (CKD) is a common problem in CKD patients, particularly in hemodialysis dependent end-stage renal disease (ESRD). Previous study showed higher mortality rate in CKD patients with anemia. ${ }^{1}$ Erythropoietin deficiency is the major essential mechanism of anemia in CKD patients. $^{2}$ The erythropoiesis stimulating agent (ESA) is the primary treatment of anemia in CKD. Clinical practice guidelines, Kidney Disease: Improving Global Outcomes (KDIGO) 2012, recommended keeping target goals for hemoglobin levels at $11-12 \mathrm{~g} / \mathrm{dL}$ in CKD patients with ESA usage. $^{3}$ Despite adequate ESA treatment, some patients remain anemic or eventually lose response to ESA, defined as ESA hyporesponsiveness. There are many plausible causes of anemia in ESA hyporesponsiveness conditions including nutritional deficiency, co-existing bone marrow diseases, and secondary hyperparathyroidism. ${ }^{2,4}$ Secondary hyperparathyroidism is also a major cause of ESA hyporesponsiveness. Pathogenesis of anemia in secondary hyperparathyroidism is still unclear but could be attributed to decreased red blood cell (RBC) production from calcitriol deficiency or the inhibition of erythropoiesis from parathyroid hormone associated with bone marrow fibrosis and interference with red cell survival. ${ }^{5}$ Previous studies in hemodialysis patients revealed a significant relationship between ESA hyporesponsiveness and higher intact parathyroid hormone (iPTH) level,,${ }^{6,7}$ and the inverse relationship between iPTH and hemoglobin level. ${ }^{8}$ One particular study demonstrated that serum iPTH level above $122 \mathrm{pg} / \mathrm{mL}$ had 67\% sensitivity and $75 \%$ specificity to predict hemoglobin level below $10.0 \mathrm{~g} / \mathrm{dL} .{ }^{9}$ Herein, we attempted to explore the causes of anemia in CKD with ESA hyporesponsiveness, in order to analyze the factors that could affect hemoglobin levels in CKD patients referred to the Hematology clinic for anemia evaluation.
Correspondence: Chantana Polprasert, Division of Hematology, Department of Medicine, Faculty of Medicine, Chulalongkorn University, King Chulalongkorn Memorial Hospital, Bangkok, Thailand.

Tel.: +6698-287-5290

E-mail: jeedchantana@gmail.com

Key words: Anemia, Chronic kidney disease, Secondary hyperparathyroidism.

Acknowledgements: We would like to express our sincere gratitude to $\mathrm{Mr}$. Preecha Reaungwechworrachai and the administrative staff of the Department of Pathology who helped in organizing specimen retrieval, preparation, and staining. This study protocol has been approved by the Research Institute's Committee on Human Research.

Contributions: KA collected, analyzed data and wrote the manuscript. NS supervised as a nephrologist's view and wrote the manuscript. $\mathrm{KW}$ analyzed data and wrote the manuscript. TA evaluated the bone marrow study. $\mathrm{CP}$ initiated, conceptualized the overall research and wrote the manuscript. All authors read and approved the final manuscript.

Conflict of interest: the authors declare no potential conflict of interests.

Funding: This research was supported by the Ratchadapiseksompoj grant (RA60-017), the Research affair, Chulalongkorn University and a grant from Research Collaborations in Hematologic Malignancies and Hematopoietic Stem Cell Transplantation.

Received for publication: 25May 2019.

Revision received: 24 July 2019.

Accepted for publication: 7 August 209

This work is licensed under a Creative Commons Attribution-NonCommercial 4.0 International License (CC BY-NC 4.0).

${ }^{(C)}$ Copyright: the Author(s), 2019

Licensee PAGEPress, Italy

Hematology Reports 2019; 11:8183

doi:10.4081/hr.2019.8183

\section{Materials and Methods}

\section{Study participants}

Our study retrospectively reviewed the data of all CKD patients referred to the Hematology clinic at King Chulalongkorn Memorial Hospital for anemia consultation from January 2007 to June 2017. All CKD patients who were deemed anemic despite 
receiving erythropoiesis stimulating agents and underwent bone marrow study were included in this analysis. Subjects with iron deficiency anemia were excluded by iron study prior to the bone marrow study.

\section{Study design}

Baseline demographic data, underlying diseases, and ESA dose per week were collected. Laboratory data within 6 months from date of bone marrow study including hemoglobin, reticulocyte count, ferritin, transferrin saturation, blood urea nitrogen (BUN), creatinine, creatinine clearance, intact parathyroid hormone, calcium, phosphate, albumin, alkaline phosphates were also recorded.

CKD was defined by having a glomerular filtration rate (GFR) $<60 \mathrm{~mL} / \mathrm{min} / 1.73$ $\mathrm{m}^{2}$ for 3 months or more. ${ }^{10}$ Patients with anemia were defined by having a hemoglobin level below $10 \mathrm{~g} / \mathrm{dL}$. ESAs were administered for at least 3 months. High parathyroid group was defined as having an iPTH level above $65 \mathrm{pg} / \mathrm{mL}$ according to the reference range of serum iPTH level at King Chulalongkorn Memorial Hospital.

\section{Bone marrow study}

Bone marrow assessments were conducted by both a hematologist and a hematopathologist. Assessments were made from aspirate smears, core biopsies and reticulin stains. Bone marrow fibrosis was semiquantitatively determined by reticulin and trichrome staining. Reticulin was graded according to the World Health Organization (WHO) as $0-3 .{ }^{11}$

\section{Statistical analysis}

For descriptive analysis, we used IBM SPSS statistics 22 program to calculate con- tinuous data including percentage, mean and standard deviation for various characteristics. To compare the normal and high parathyroid groups, we analyzed differences by conducting independent t-test with a significant P-value at 0.05 using IBM SPSS statistics 22 program. Moreover, univariate analysis using a Pearson correlation coefficient and multivariate analysis were used for correlation between hemoglobin levels and other variable data including age, gender, body mass index (BMI), iPTH, serum calcium, phosphate, serum albumin, transferrin saturation ratio, ferritin, hemodialysis and non-hemodialysis cases, using Strata version 15.1 program.

\section{Results}

\section{Patient's characteristics}

A total of 87 patients were included in the study and all patients were performed a bone marrow study to investigate the causes of anemia. Eleven patients (12.6\%) were diagnosed as having bone marrow disease [8 pure red cell aplasia (PRCA) and 3 myelodysplastic syndromes (MDS)], while three cases had thalassemia diseases. Because serum iPTH were not available in 30 patients, only 43 cases could be evaluated for mineral status.

Among the 43 CKD patients, the mean age was 63 (range 33-88) years old and $54.5 \%$ were male. The common coexisting diseases included hypertension $(\mathrm{n}=30$; $69.8 \%)$, diabetes mellitus $(n=16 ; 37.2 \%)$ and dyslipidemia $(\mathrm{n}=13 ; \quad 30.2 \%)$. Underlying causes of chronic kidney disease were diabetic nephropathy, chronic glomerulonephritis, hypertensive nephropathy in $14(32.6 \%), 4(9.3 \%)$ and $1(2.3 \%)$ cases, respectively. Thirty-six $(83.7 \%)$ patients were on chronic hemodialysis. Evaluation for bone marrow fibrosis by reticulin staining was performed in 42 patients with chronic kidney disease (Table 1). Most of the patients (29/42) showed fibrosis grade 1 . In addition, 4 patients showed fibrosis grade 3-4 and grade 2. Three patients showed fibrosis grade 0 .

\section{Potential factors associated with ESA hyporesponsiveness}

The mean hemoglobin $(\mathrm{Hb})$ level from the $43 \mathrm{CKD}$ patients was $8.49 \mathrm{~g} / \mathrm{dL}( \pm 1.19)$. Thirty-four $(79.1 \%)$ patients showed high intact parathyroid (iPTH) level (iPTH $\geq 65$ $\mathrm{pg} / \mathrm{mL}$ ). Mean iPTH levels were found to be $361.73 \mathrm{pg} / \mathrm{mL}$ (22.9-3,362). Clinical characteristics of patients with high and normal iPTH level are shown in Table 2. Patients with high iPTH levels were older than those with normal iPTH levels (71 vs. 60 years old; $\mathrm{P}=0.02$ ). Comparing normal and high iPTH groups, a significantly lower hemoglobin level (8.29 vs $9.24 \mathrm{mg} / \mathrm{dL} ; \mathrm{P}=0.032)$ and a higher dose of erythropoietin usage (16,352.94 vs 12,444.44 unit/week; $\mathrm{P}=0.024)$ were demonstrated in the high iPTH group (Table 3). In addition, serum calcium was found to be significantly lower in patients with high iPTH compared to normal iPTH levels (9.33 vs. $10.25 \mathrm{mg} / \mathrm{dL}$;

Table 1. Fibrosis grading by reticulin staining.

Fibrosis grade Number (total $\mathrm{N}=42$ )

\begin{tabular}{lc}
0 & 3 \\
1 & 29 \\
\hline $1-2$ & 4 \\
2 & 4 \\
\hline
\end{tabular}

Table 2. Clinical characteristics of whole, normal iPTH and high iPTH groups.

\begin{tabular}{lccc} 
Variables & All patients & $\begin{array}{c}\text { Normal iPTH } \\
(<65 \mathrm{pg} / \mathrm{mL})\end{array}$ & $\begin{array}{c}\text { High iPTH } \\
(>65 \mathrm{pg} / \mathrm{mL})\end{array}$ \\
No. Patients & $43(100 \%)$ & $9(20.9 \%)$ & $34(79.1 \%)$ \\
Age (years) & $63.0(33.0-88.0)$ & $70.22(60.0-86.0)$ & $61.09(33.0-88.0)$ \\
\hline Sex (\%) & $24(55.8)$ & $5(55.6)$ & $19(55.9)$ \\
Male & $19(44.2)$ & $4(44.4)$ & $15(44.1)$ \\
Female & $21.29(14.20-26.45)$ & $22.30(21.6-23.2)$ & $21.18(14.2-26.45)$ \\
BMI (Kg/m $\left.{ }^{2}\right)$ & $16(37.2)$ & & $12(35.3)$ \\
\hline Underlying disease (\%) & $30(69.8)$ & $8(44.4)$ & $22(22)$ \\
Diabetes & $13(30.2)$ & $3(38.9)$ & $10(29.4)$ \\
Hypertension & $6(14.0)$ & $1(11.1)$ & $5(14.7)$ \\
Dyslipidemia & $36(83.7)$ & $8(88.9)$ & $28(82.4)$ \\
No underlying & & & - \\
ESRD on HD (\%) & & & - \\
\hline
\end{tabular}


$\mathrm{P}=0.025$ ). In a univariate analysis followed by a stepwise multivariate analysis, serum phosphate was significantly associated with lower hemoglobin level $(\mathrm{P}=0.01$ and $\mathrm{P}=0.013$, respectively, Table 4). As shown in Figure 1, serum hemoglobin level was inversely correlated with $\mathrm{PPTH}$ and serum phosphate level $(\mathrm{r}=-0.54, \mathrm{P}<0.001$ and $\mathrm{r}=$ $0.47, \mathrm{P}=0.005$; respectively). When classifying iPTH levels into 1 to more than 9 times of the normal level, 6 patients $(14 \%)$ had a serum $\mathrm{PPTH}$ nine times higher than the normal level. Patients with iPTH three times more than the normal value $(n=4)$ showed significantly lower hemoglobin levels than patients with iPTH three times lower than the normal value $(\mathrm{n}=27),(\mathrm{Hb} 7.3$ $\mathrm{g} / \mathrm{dL}$ vs. $9 \mathrm{~g} / \mathrm{dL}, \mathrm{P}=0.01$ ).

\section{Discussion}

This study was done in CKD patients with anemia who were already treated with erythropoiesis stimulating agents (ESA). Iron deficiency anemia was excluded by iron study and positive iron staining in bone marrow. Bone marrow disease was the cause of anemia in $12 \%(11 / 87)$ of subjects, while $3.4 \%$ (3/87) were diagnosed as thalassemia diseases. In patients with normal bone marrow study, patients with higher iPTH level had lower hemoglobin level than patients with a normal iPTH level. This result was concordant with the previous

Table 3. Biochemistry of whole, normal iPTH and high iPTH groups.

\begin{tabular}{|c|c|c|c|c|}
\hline Variables & All patients & Normal iPTH & $\begin{array}{l}\text { High iPTH } \\
(<65 \mathrm{pg} / \mathrm{mL})\end{array}$ & $\begin{array}{c}P \\
(>65 \mathrm{pg} / \mathrm{mL})\end{array}$ \\
\hline No. Patients & $43(100 \%)$ & $9(20.9 \%)$ & $34(79.1 \%)$ & - \\
\hline $\mathrm{Hb}(\mathrm{g} / \mathrm{dL})$ & $8.49( \pm 1.19)$ & $9.24( \pm 0.61)$ & $8.29( \pm 1.24)$ & 0.032 \\
\hline Iron (microgram/dL) & $82.1( \pm 48.26)$ & $89.22( \pm 60.9)$ & $80.06( \pm 45.12)$ & 0.623 \\
\hline Ferritin $(\mathrm{ng} / \mathrm{mL})$ & $1115.84( \pm 895.21)$ & $1162.32( \pm 1006.0)$ & $1102.77( \pm 878.68)$ & 0.863 \\
\hline Transferrin (mg/dL) & $215.9( \pm 65.80)$ & $231.22( \pm 101.08)$ & $211.45( \pm 53.05)$ & 0.586 \\
\hline Transferrin saturation (\%) & $40.87( \pm 25.96)$ & $42.23( \pm 28.23)$ & $40.47( \pm 25.74)$ & 0.861 \\
\hline Reticulocyte count & $74050( \pm 35193.49)$ & $69000( \pm 22068.07)$ & $74771.43( \pm 37031.03)$ & 0.79 \\
\hline Calcium (mg/dL) & $9.50( \pm 0.99)$ & $10.25( \pm 0.85)$ & $9.33( \pm 0.95)$ & 0.025 \\
\hline Phosphate (mg/dL) & $4.45( \pm 2.18)$ & $4.08( \pm 1.58)$ & $4.52( \pm 2.29)$ & 0.656 \\
\hline ALP (IU/L) & $122.72( \pm 85.48)$ & $84.2( \pm 27.04)$ & $134.05( \pm 93.84)$ & 0.261 \\
\hline Albumin (mg/dL) & $3.51( \pm 0.61)$ & $3.20( \pm 0.66)$ & $3.5( \pm 0.58)$ & 0.141 \\
\hline ESA dose (unit/week) & $15534.88( \pm 7983.16)$ & $12444.44( \pm 2185.81)$ & $16352.94( \pm 8755.54)$ & 0.024 \\
\hline Iron supplement & $16(37.2 \%)$ & $4(44.4 \%)$ & $12(35.3 \%)$ & - \\
\hline
\end{tabular}

Table 4. Univariate and multivariate analysis.

\begin{tabular}{|c|c|c|c|c|}
\hline Parameters & & & Stepwise I & \\
\hline & Coefficient $(95 \% \mathrm{CI})$ & P & Coefficient $(95 \%$ CI) & $\mathbf{P}$ \\
\hline Age & 0.023 (0.00 to 0.046$)$ & 0.05 & & \\
\hline BMI & $0.067(-0.09$ to 0.22$)$ & 0.37 & & \\
\hline Serum iPTH/1000 & $-0.53(-1.07$ to 0.002$)$ & 0.05 & & \\
\hline Calcium & 0.086 (-0.309 to 0.48$)$ & 0.66 & & \\
\hline Serum phosphate & $-0.22(-0.38$ to -0.05$)$ & 0.01 & $-0.22(-0.38$ to -0.049$)$ & 0.013 \\
\hline Albumin & $-0.24(-0.91$ to 0.43$)$ & 0.47 & & \\
\hline
\end{tabular}

In multivariate adjusting for age and serum iPTH and using stepwise for selected final model.

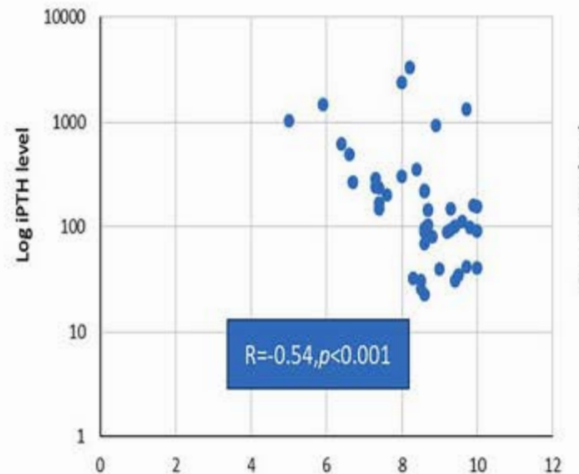

A
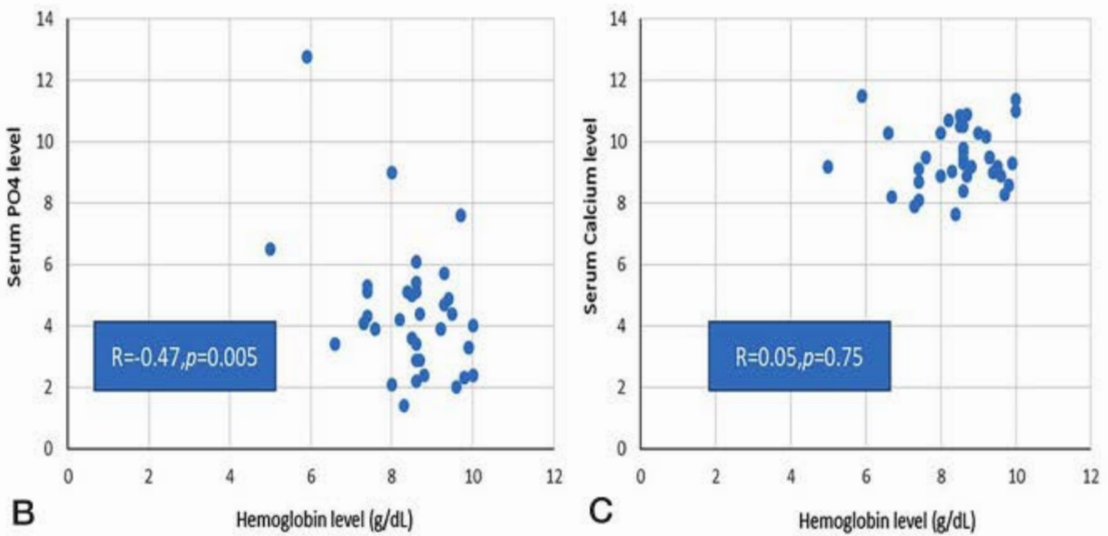

Figure 1. Correlation of Hemoglobin with serum iPTH (A), serum phosphate (PO4) (B) and serum Calcium (C). 
studies. ${ }^{12,13}$ Association of secondary hyperparathyroidism and anemia in chronic kidney disease which could be explained from the inhibitory effect of parathyroid hormone to erythropoietin secretion corresponded with a study showing higher serum erythropoietin level after parathyroidectomy. ${ }^{14}$ Besides the above-mentioned mechanisms, some studies demonstrated the inhibitory effect of parathyroid hormone on the growth of bone marrow erythroid progenitors and stimulation of fibrous formation in bone marrow. ${ }^{15}$ But, in our study there was no significant bone marrow fibrosis. A previous study also showed that treatment of secondary hyperparathyroidism with cinacalcet (calcimimetic) could increase hemoglobin levels in chronic hemodialysis patients. ${ }^{16}$ In non-hemodialysis CKD, optimal iPTH level is not known, but maintaining a normal iPTH level is recommended. Although the target level of intact parathyroid hormone in hemodialysis dependent ESRD patients within two to nine times above normal value is suggested, ${ }^{17}$ we found that patients with a serum iPTH level higher than three times above upper normal limit showed lower hemoglobin levels than patients with elevated iPTH level less than three times above normal levels. This finding might indirectly suggest the optimal iPTH level in CKD patients in order to correct anemia associated with EPO hyporesponsiveness.

Due to evidence of worse outcome in hyperphosphatemia, the KDIGO guideline in 2017 recommended to lower phosphate levels to normal range in both non-dialysis and dialysis CKD. ${ }^{17}$ Our study showed that high serum phosphate was an independent factor of low hemoglobin level. This fact supported phosphate lowering treatment in CKD patients. The strength of our study came from the fact that all patients underwent bone marrow studies to exclude bone marrow disease. However, this study had some limitations as several patients were excluded due to missing iPTH values and hepcidin, as one of the major players of anemia in CKD was not evaluated, which was one of the players in anemia in CKD. Our study demonstrated disturbance of mineral equilibrium and hyperparathyroidism as major causes of ESA hyporesponsive anemia, after excluding iron deficiency anemia and bone marrow diseases. In this study, we didn't show data of drug or treatment such as activated vitamin D and calcimimetics that could affect calcium and phosphate metabolism. By the result of serum phosphate and calcium level whether receive those treatment or not, we could implement the results of our study by promoting inten- sive phosphate lowering treatment in order to achieve normal serum phosphate levels and lower serum iPTH levels not to be higher than three times above normal range as initial steps of anemia management in CKD before considering bone marrow study in this patient population. A prospective follow-up study might be helpful to support the hypothesis.

\section{Conclusions}

In conclusion, this study showed that hyperphosphatemia was significantly associated with lower hemoglobin level in CKD-patients. Serum phosphate and serum iPTH were inversely correlated with hemoglobin levels. Patients with an iPTH level within three times above normal values had a higher hemoglobin level compared to CKD with an elevated iPTH more than three times above normal ranges. These findings strongly suggest clinicians to consider closely monitoring serum phosphate and iPTH levels as a part of anemia treatment in CKD patients before proceeding to perform bone marrow study.

\section{References}

1. Snyder JJ, Foley RN, Gilbertson DT, et al. Collins AJ. Hemoglobin levels and erythropoietin doses in hemodialysis and peritoneal dialysis patients in the United States. J Am Soc Nephrol 2004;15:174-9.

2. Fishbane S, Spinowitz B. Update on anemia in ESRD and earlier stages of CKD: Core Curriculum 2018. AJKD 2018;71:423-35.

3. Kidney Disease: Improving Global Outcomes (KDIGO) Anemia Work Group. KDIGO Clinical Practice Guideline for Anemia in Chronic Kidney Disease. Kidney Int Suppl 2012;2:279-335.

4. Kanbay M, Perazella MA, Kasapoglu B, et al. Erythropoiesis stimulatory agent-resistant anemia in dialysis patients: review of causes and management. Blood Purif 2010;29:1-12.

5. Drüeke TB, Eckardt KU. Role of secondary hyperparathyroidism in erythropoietin resistance of chronic renal failure patients. Nephrol Dial Transplant 2002;17:28-31.

6. Al-Hilali N, Al-Humoud H, Ninan VT, et al. Does parathyroid hormone affect erythropoietin therapy in dialysis patients? Med Princ Pract 2007;16:637.

7. Rao DS, Shih MS, Mohini R. Effect of Serum Parathyroid Hormone and Bone Marrow Fibrosis on the Response to Erythropoietin in Uremia. N Engl J Med 1993;328:171-5.

8. Chutia H, Ruram AA, Bhattacharyya $\mathrm{H}$, et al. Association of secondary hyperparathyroidism with hemoglobin level in patients with chronic kidney disease. J Lab Physicians 2013;5:51-4.

9. Russo D, Morrone L, Di Iorio B, et al. Parathyroid hormone may be an early predictor of low serum hemoglobin concentration in patients with not advanced stages of chronic kidney disease. J Nephrol 2015;28:701-8.

10. Levey AS, Eckardt K, Tsukamoto Y, et al. Definition and classification of chronic kidney disease: A position statement from Kidney Disease: Improving Global Outcomes (KDIG). Kidney International 2005;67:2089-100.

11. Swerdlow SH, Campo E, Harris NL, Jaffe ES. WHO Classification of Tumours of Haematopoietic and Lymphoid Tissues, 4th ed. Geneva: World Health Organization; 2017.

12. Baradaran A, Nasri H. Intensification of anemia by secondary hyperparathyroidism in hemodialysis patients. Med J Islamic World Acad Sci 2001;14:161-6.

13. Ahamed AAI, Salih KA, Abderahaman YA, et al. Relationship between anemia and parathyroid disorders in hemodialysis patients. Eur Acad Res 2016;8:652735.

14. Urena P, Eckardt KU, Sarfati E. Serum erythropoietin and erythropoiesis in primary and secondary hyperparathyroidism: effect of parathyroidectomy. Nephron 1991;59:384-93.

15. Gallieni M, Corsi C, Brancaccio D. Hyperparathyroidism and Anemia in Renal Failure. Am J Nephrol 2000;20:89-96.

16. Mpio I, Boumendjel N, Karaaslan H, et al. Secondary hyperparathyroidism and anemia. Effects of a calcimimetic on the control of anemia in chronic hemodialysed patients. Pilot Study. Nephrol Ther 2011;7:229-36.

17. Kidney Disease: Improving Global Outcomes (KDIGO) CKD-MBD Update Work Group. KDIGO 2017 clinical practice guideline update for the diagnosis, evaluation, prevention, and treatment of chronic kidney diseasemineral and bone disorder (CKDMBD). Kidney Int Suppl 2017;7:1-59. 